\title{
Analysis of Normalized Point Source Sensitivity as a performance metric for the Thirty Meter Telescope
}

Byoung-Joon Seo, Carl Nissly, George Angeli, Brent Ellerbroek, Jerry Nelson, et al.

Byoung-Joon Seo, Carl Nissly, George Angeli, Brent Ellerbroek, Jerry Nelson, Norbert Sigrist, Mitchell Troy, "Analysis of Normalized Point Source Sensitivity as a performance metric for the Thirty Meter Telescope," Proc. SPIE 7017, Modeling, Systems Engineering, and Project Management for Astronomy III, 70170T (11 July 2008); doi: 10.1117/12.790453

Event: SPIE Astronomical Telescopes + Instrumentation, 2008, Marseille, France 


\title{
Analysis of Normalized Point Source Sensitivity as a performance metric for the Thirty Meter Telescope
}

\author{
Byoung-Joon Seo ${ }^{a, b}$, Carl Nissly ${ }^{a, b}$, George Angeli ${ }^{c}$, Brent Ellerbroek ${ }^{c}$, Jerry Nelson $^{c}$, \\ Norbert Sigrist $^{a, b}$, and Mitchell Troy ${ }^{a, b}$ \\ ${ }^{a}$ Jet Propulsion Laboratory, Pasadena, CA. 91109, USA \\ ${ }^{b}$ California Institute of Technology, CA. 91125, USA \\ ${ }^{c}$ Thirty Meter Telescope Observatory, CA. 91107, USA
}

\begin{abstract}
We investigate a new metric, Normalized Point Source Sensitivity (PSSN), for characterizing the seeing limited performance of the Thirty Meter Telescope. As the PSSN metric is directly related to the photometric error of background limited observations, it truly represents the efficiency loss in telescope observing time. The PSSN metric properly accounts for the optical consequences of wavefront spatial frequency distributions due to different error sources, which makes it superior to traditional metrics such as the $80 \%$ encircled energy diameter. We analytically show that multiplication of individual PSSN values due to individual errors is a good approximation for the total PSSN when various errors are considered simultaneously. We also numerically confirm this feature for Zernike aberrations, as well as for the numerous error sources considered in the TMT error budget using a ray optics simulator, Modeling and Analysis for Controlled Optical Systems. We also discuss other pertinent features of the PSSN including its relations to Zernike aberration and RMS wavefront error.
\end{abstract}

Keywords: Point Source Sensitivity, Optical Modeling, Image Plane Metric, MACOS, Thirty Meter Telescope

\section{INTRODUCTION}

It is a goal of Thirty Meter Telescope (TMT) that seeing limited performance of the telescope achieves $90 \%$ of that of a perfect telescope. "Performance" (or science) is an abstract term and can be represented using a performance metric. Therefore, a performance metric should describe performance (science) well. In addition, it is desirable to predict or estimate overall performance when aberrations from multiple error sources are considered. This estimation feature of a metric is even more important when designing and budgeting errors for a large telescope such as TMT since its modeling tasks are highly distributed to multiple optical designers, vendors and manufacturers. ${ }^{1}$

The root mean square of the wavefront error (RMS WFE) and 80\% encircled energy diameter (EE80) are often used as a performance metrics. The RMS WFE is useful when estimation of multiple aberration errors is needed since overall RMS WFE is simply the square root of the sum of the square (RSS) of RMS WFEs of individual aberrations. However, the RMS WFE fails in describing telescope's performance well enough since a higher spatial frequency aberration degrades the science more than lower frequency aberrations even though they both have the same RMS WFE. On the other hand, the EE80 is known to describe the science well but overall estimation from multiple error sources is challenging since it has non-linear characteristics when errors are in the physical optics regime.

We investigate a new metric, Normalized Point Source Sensitivity (PSSN), for characterizing the seeing limited performance of the TMT. As the PSSN metric is directly related to the photometric error of background limited observations, it accurately represents the efficiency loss in telescope observing time. The PSSN metric properly accounts for the optical consequences of wavefront spatial frequency distributions due to different error sources, which makes it superior to traditional metrics such as RMS WFE and EE80.

Further author information: (Send correspondence to Byoung-Joon Seo)

Byoung-Joon Seo: E-mail: Byoung-Joon.Seo@jpl.nasa.gov

Modeling, Systems Engineering, and Project Management for Astronomy III, edited by George Z. Angeli,

Martin J. Cullum, Proc. of SPIE Vol. 7017, 70170T, (2008) · 0277-786X/08/\$18 · doi: 10.1117/12.790453 


\section{ANALYTICAL ANALYSIS OF NORMALIZED POINT SOURCE SENSITIVITY}

\subsection{Analytical Definition of Normalized Point Source Sensitivity}

The metric, Point Source Sensitivity (PSS), is simply the integration of the square of the point spread function (PSF) over the entire image space and $\mathrm{King}^{2}$ found that the PSS is a metric that maximizes the signal to noise ratio (SNR) of images formed on a digital detector array. In order to reflect telescope performance in terms of science ratio, we propose the normalized PSS (PSSN), which represents the degradation ratio of the aberrated PSS to the ideal PSS in the seeing limited condition. Therefore, the proposed metric, PSSN, can be denoted as,

$$
P S S N=\frac{\int_{\infty}\left|P S F_{\text {err }}(\vec{\theta})\right|^{2} d \vec{\theta}}{\int_{\infty}\left|P S F_{o}(\vec{\theta})\right|^{2} d \vec{\theta}}
$$

where $P S F_{o}$ is the time-averaged PSF of the ideal telescope exposed to the atmosphere and $P S F_{\text {err }}$ is the time-averaged PSF of an aberrated telescope exposed to the atmosphere. The PSF is defined over $\vec{\theta}$, which can be in angular space or spatial units.

Since the optical transfer function (OTF) and PSF are in Fourier transformation relations and according to the Parseval's energy conservation theorem, Eq. (1) can be represented using OTFs,

$$
P S S N=\frac{\int_{\infty}\left|O T F_{\text {err }}(\vec{f})\right|^{2} d \vec{f}}{\int_{\infty}\left|O T F_{o}(\vec{f})\right|^{2} d \vec{f}}
$$

where $O T F_{o}$ and $O T F_{\text {err }}$ are the OTF of $P S F_{o}$ and $P S F_{\text {err }}$, respectively. The OTF has coordinate's $\vec{f}$ in the units of cycles per radian or meter.

For aberrations whose the optical path difference (OPD) has random and shift-invariant characteristics, we find in Appendix A that $O T F_{e r r}$ can be represented as multiplication of $O T F_{o}$ and $O T F_{e}$, where $O T F_{e}$ is the time-averaged OTF due to the telescope aberrations only, i.e.: $O T F_{\text {err }}(\vec{f})=O T F_{e}(\vec{f}) O T F_{o}(\vec{f})$. Then, $P S S N$ in Eq. (2) can be further decomposed as,

$$
P S S N=\left\langle\left|O T F_{e}(\vec{f})\right|^{2}\right\rangle
$$

where $\langle\cdot\rangle$ defines a new operator, average of atmosphere, such that,

$$
\langle\cdot\rangle \equiv \frac{\int_{\infty}(\cdot)\left|O T F_{o}(\vec{f})\right|^{2} d \vec{f}}{\int_{\infty}\left|O T F_{o}(\vec{f})\right|^{2} d \vec{f}}
$$

We use this operator for the remainder of this paper for random shift-invariant aberrations. Note that the operator is linear, i.e. $\langle A+B\rangle=\langle A\rangle+\langle B\rangle$, and mathematically similar in many ways to the average operator used in the random variable theory with the $\left|O T F_{o}\right|^{2}$ being a probability density function. Thus, mathematically, PSSN is the average of $\left|O T F_{e}\right|^{2}$ over $\left|O T F_{o}\right|^{2}$.

\subsection{Properties of Normalized Point Source Sensitivity}

Based on the definition, we find that the PSSN has following properties, which we discuss in the remainder of this section.

$\boldsymbol{P} 1$ The PSSN becomes unity when there are no telescope errors and becomes 0 when the error is infinitely large.

$$
0 \leq P S S N \leq 1
$$

P2 The PSSN has a multiplicative feature for low frequency and weak aberrations. Suppose there are multiple errors on the telescope. Let $P S S N_{\text {combined }}$ and $P S S N_{\text {multiplied }}$ be the PSSN for all combined error and for multiplied values of PSSN from individual errors, respectively. Then, $P S S N_{\text {multiplied }}$ approximates PSSN $N_{\text {combined }}$.

$$
P S S N_{\text {combined }} \approx P S S N_{\text {multiplied }}
$$


P3 The PSSN has a relation to RMS WFE if we assume the RMS WFE is small.

$$
P S S N \approx 1-\alpha \sigma^{2}
$$

where $\sigma$ is the RMS WFE and $\alpha$ is a proportional constant independent to $\sigma$. However, $\alpha$ is a function of aberration frequency (correlation length of OPD) and atmosphere $r_{o}$.

$P 4$ The PSSN can be approximated to the square of the Strehl for white noise aberration ( 0 correlation length of OPD). This is due to that the energy in PSF spreads out beyond atmosphere $r_{o}$. Therefore,

$$
P S S N \approx 1-2 \sigma^{2}
$$

where $\sigma$ is the RMS WFE in radians and $\alpha$ becomes 2 .

\subsubsection{Multiplicative features}

First, we make the following assumptions regarding the PSSN in the analytical studies of multiplicative features.

A1 Normalized Power: We assume the total power on the aperture is unity. All OTF's have a value of one at the origin.

A2 Random and shift-invariant aberration: We assume that aberrated OPDs have random and shift-invariant characteristics (See Appendix A for its mathematical definition). This assumption allows us to use Eq. (3).

A3 Low order and weak aberration: In all errors on TMT, we observe that the $O T F_{e}(\vec{f})$ is much wider than the $O T F_{o}(\vec{f})$. This implies that $O T F_{e}(\vec{f})$ changes only by small amount over the region of interest where $O T F_{o}(\vec{f})$ is defined meaningfully. Therefore, (for calculation convenience) we define a small valued positive real function, $\epsilon(\vec{f})$ such that,

$$
\left|O T F_{e}(\vec{f})\right|^{2}=1-\epsilon(\vec{f})
$$

As the error becomes smaller or the correlation length of OPD due to the error becomes larger than the atmosphere correlation length, $r_{o}$, the $\epsilon(\vec{f})$ becomes small relative to unity near the origin.

Suppose that there are two different errors on the telescope, which generate their $\left|O T F_{e}(\vec{f})\right|^{2} \mathrm{~s}$ as $1-\epsilon_{1}(\vec{f})$ and $1-\epsilon_{2}(\vec{f})$. Since the total OTF is a product of two individual OTFs and the averaging operator is linear, the combined PSSN, PSSN $N_{\text {combined }}$, is computed as,

$$
\begin{aligned}
P S S N_{\text {combined }} & =\left\langle\left(1-\epsilon_{1}\right)\left(1-\epsilon_{2}\right)\right\rangle \\
& =1-\left\langle\epsilon_{1}\right\rangle-\left\langle\epsilon_{2}\right\rangle+\left\langle\epsilon_{1} \epsilon_{2}\right\rangle
\end{aligned}
$$

where we denote $\epsilon_{i}(\vec{f})$ as $\epsilon_{i}$ for simplicity. On the other hand, the multiplied value PSSN $N_{\text {multiplied }}$ of PSSNs from individual PSSN can be denoted as,

$$
\begin{aligned}
P S S N_{\text {multiplied }} & =\left\langle 1-\epsilon_{1}\right\rangle\left\langle 1-\epsilon_{2}\right\rangle \\
& =1-\left\langle\epsilon_{1}\right\rangle-\left\langle\epsilon_{2}\right\rangle+\left\langle\epsilon_{1}\right\rangle\left\langle\epsilon_{2}\right\rangle
\end{aligned}
$$

These two equations immediately imply that PSSN is multiplicative to first order since the first orders, $\left\langle\epsilon_{i}\right\rangle$, are small according to $A 3$, thus, their second orders are even smaller. In order to further investigate the PSSN multiplicative feature beyond the first order, we define the difference of Eq. (10) and Eq. (11) as $\Delta_{1,2}$.

$$
\Delta_{1,2}=\left\langle\epsilon_{1} \epsilon_{2}\right\rangle-\left\langle\epsilon_{1}\right\rangle\left\langle\epsilon_{2}\right\rangle
$$

Note that $\Delta_{1,2}$ is mathematically similar to the statistic correlation in the random probability theory. According to the theory, $\Delta_{1,2}$ is bounded by the product of the standard deviations of $\epsilon_{1}(\vec{f})$ and $\epsilon_{2}(\vec{f})$,

$$
\left|\Delta_{1,2}\right| \leq \sqrt{\left(\left\langle\epsilon_{1}^{2}\right\rangle-\left\langle\epsilon_{1}\right\rangle^{2}\right)\left(\left\langle\epsilon_{2}^{2}\right\rangle-\left\langle\epsilon_{2}\right\rangle^{2}\right)}
$$


and becomes 0 when two $\epsilon_{i}(\vec{f})$ are uncorrelated.

Assuming $A 3$, we can further calculate the $\Delta_{1,2}$ in Eq. (12) using the Taylor expansion. We approximate the $\epsilon_{i}(\vec{f})$ up to the second order Taylor expansion coefficient after assuming the aberrations are isotropic. i.e $\epsilon_{i}(\vec{f})$ is only a function of magnitude of $\vec{f}$. Due to mathematical complexity, we detail the calculation in Appendix B.

$$
\Delta_{1,2} \approx 1.2119\left(1-P S S N_{1}\right)\left(1-P S S N_{2}\right)
$$

where $P S S N_{1}$ and $P S S N_{2}$ are individual PSSN of two error sources and the constant, 1.2119, represents a dimensionless unique value for the case of atmosphere we applied. By using Taylor series approximation up to the second order, note that $\Delta_{1,2}$ is a positive number and is equal to the maximum boundary in Eq. (13). Since we use the same positive polarity of coefficients for approximating $\epsilon_{1}(\vec{f})$ and $\epsilon_{2}(\vec{f})$, the result obtained in Eq. (14) represents the correlation coefficient of 1, thus, is same as the analytical boundary in Eq. (13). However, in general, $\Delta_{1,2}$ can be either positive or negative depending on the correlation between two $\epsilon_{i}(\vec{f})$. Therefore, Eq. (14) is reformulated using analytical boundary $\Delta_{A}$.

$$
\left|\Delta_{1,2}\right| \leq \Delta_{A} \approx 1.2119\left(1-P S S N_{1}\right)\left(1-P S S N_{2}\right)
$$

Eq. (15) implies that the difference $\Delta_{1,2}$ between $P S S N_{\text {combined }}$ and $P S S N_{\text {multiplied }}$ is within the analytical boundary, $\Delta_{A}$. i.e:

$$
\left|P S S N_{\text {combined }}-P S S N_{\text {multiplied }}\right| \leq \Delta_{A}
$$

Note that $1-P S S N_{i}$ is normally a small value, thus $\Delta_{A}$ is on the order of the square of $1-P S S N_{i}$. For example, let us assume that $P S S N_{1}=P S S N_{2}=0.95$. Then, $\Delta_{A}$ becomes $3 \times 10^{-3}$.

Furthermore, we consider $N$ different errors by generalizing Eq. (12). Suppose that individual errors generate $\epsilon_{i}$ where $i=1,2, \cdots, N$ and their multiplied and combined PSSN are PSSN $N_{\text {multiplied }}$ and $P S S N_{\text {combined }}$, respectively. Then, the difference, $\Delta_{T}$, between multiplied and combined PSSN becomes:

$$
\begin{aligned}
\Delta_{T} & =\sum_{i=1}^{N} \sum_{j=i+1}^{N}\left(\left\langle\epsilon_{i} \epsilon_{j}\right\rangle-\left\langle\epsilon_{i}\right\rangle\left\langle\epsilon_{j}\right\rangle\right) \\
& -\sum_{i=1}^{N} \sum_{j=i+1}^{N} \sum_{k=j+1}^{N}\left(\left\langle\epsilon_{i} \epsilon_{j} \epsilon_{k}\right\rangle-\left\langle\epsilon_{i}\right\rangle\left\langle\epsilon_{j}\right\rangle\left\langle\epsilon_{k}\right\rangle\right)+\cdots \\
& +(-1)^{N}\left(\left\langle\epsilon_{1} \epsilon_{2} \epsilon_{3} \cdots \epsilon_{N}\right\rangle-\left\langle\epsilon_{i}\right\rangle\left\langle\epsilon_{j}\right\rangle\left\langle\epsilon_{k}\right\rangle \cdots\left\langle\epsilon_{N}\right\rangle\right)
\end{aligned}
$$

We assume $A 3$ again to get an approximated boundary $\Delta_{A}$ of $\Delta_{T}$ using the Taylor expansion again as in Appendix B.

$$
\begin{aligned}
\left|\Delta_{T}\right| \leq \Delta_{A} & \approx \frac{\left\langle r^{4}\right\rangle-\left\langle r^{2}\right\rangle^{2}}{\left\langle r^{2}\right\rangle^{2}} \sum_{i=1}^{N} \sum_{j=i+1}^{N}\left(1-P S S N_{i}\right)\left(1-P S S N_{j}\right) \\
& -\frac{\left\langle r^{6}\right\rangle-\left\langle r^{2}\right\rangle^{3}}{\left\langle r^{2}\right\rangle^{3}} \sum_{i=1}^{N} \sum_{j=i+1}^{N} \sum_{k=j+1}^{N}\left(1-P S S N_{i}\right)\left(1-P S S N_{j}\right)\left(1-P S S N_{k}\right)+\cdots \\
& +(-1)^{N} \frac{\left\langle r^{2 N}\right\rangle-\left\langle r^{2}\right\rangle^{N}}{\left\langle r^{2}\right\rangle^{N}} \prod_{i=1}^{N}\left(1-P S S N_{i}\right)
\end{aligned}
$$

where $P S S N_{i}$ are the PSSN value for the $i^{\prime}$ th error. We further assume that the errors have the same PSSN (uniformly distributed errors in PSSN value sense), then, we have approximated $\Delta_{A}$ as in Eq. (19).

$$
\Delta_{A} \approx \sum_{n=2}^{N}(-1)^{n} \frac{\left\langle r^{2 n}\right\rangle-\left\langle r^{2}\right\rangle^{n}}{\left\langle r^{2}\right\rangle^{n}} \frac{(N-n) ! n !}{N !}\left(1-{ }^{N} \sqrt{1-P S S N_{\text {multiplied }}}\right)^{n}
$$




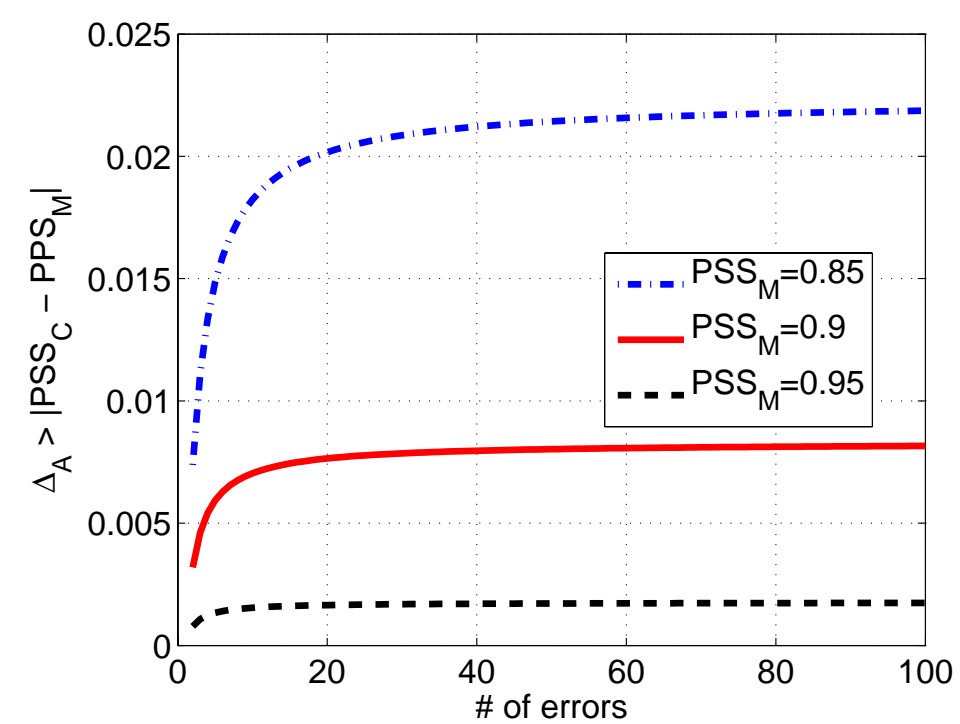

Figure 1. The analytically calculated $\Delta_{A}$ using Eq. (19) as a function of number of errors $(N)$ when the multiplied PSSNs are $0.85,0.9$ and 0.95 , respectively. $\Delta_{A}$ represents the analytical boundary for difference between combined and multiplied PSSN. We assume that the errors have the same PSSN (uniformly distributed errors in PSSN value sense). We also assume $A 2$ and $A 3$.

Fig. 1 show the analytically calculated $\Delta_{A}$ using Eq. (19) as a function of number of errors $(N)$ when the multiplied PSSNs are 0.85, 0.9 and 0.95 , respectively. When the multiplied PSSN is 0.9 , the difference, $\Delta_{A}$ is near $3 \times 10^{-3}$ and increases to around $8 \times 10^{-3}$ as the number of errors increases from 2 to 100 . This implies that the multiplicative feature is valid within $1 \%$ when the science loss of all combined errors is smaller than $10 \%$.

\subsubsection{Relation to Wavefront Errors}

We derive a general formula for $O T F_{e r r}$ from an OPD in Eq. (36) in Appendix A. We write Eq. (36) here again for convenience.

$$
O T F_{e r r}(\vec{f})=O T F_{A}(\vec{f}) \cdot \int_{r} A(\vec{r}) A(\vec{r}-\lambda \vec{f}) E\left[e^{-j k(O P D(\vec{r})-O P D(\vec{r}-\lambda \vec{f})}\right] d \vec{r}
$$

where $\vec{r}$ and $d \vec{r}$ are the two-dimensional coordinates for the OPD (typically in meter units), $\vec{f}$ is the coordinate for the OTF space (typically in cycles per radian), $k$ is the wavenumber defined as $2 \pi / \lambda, \lambda$ is the wavelength, $A(\vec{r})$ is the real, nonnegative telescope pupil function normalized with $\int A^{2}(\vec{r}) d \vec{r}=1 . O T F_{A}(\vec{f})$ is the time-averaged OTF of the atmosphere and the operator, $E[\cdot]$, denotes the time average (or statistic average) of the aberration.

To investigate the PSSN dependency on the RMS WFE, we first normalize the OPD such that

$$
O P D(\vec{r})=\sigma S(\vec{r})
$$

where $\sigma$ represents the RMS WFE in radians and $S(\vec{r})$ is the normalized OPD, i.e. $\int A(\vec{r}) S(\vec{r})^{2} d \vec{r}=1$. After denoting $\Delta S(\vec{r}, \lambda \vec{f}) \equiv S(\vec{r})-S(\vec{r}-\lambda \vec{f})$, Eq. (20) is represented as a function of RMS WFE, $\sigma$.

$$
O T F_{\text {err }}(\vec{f})=O T F_{A}(\vec{f}) \cdot \int_{r} A(\vec{r}) A(\vec{r}-\lambda \vec{f}) E\left[e^{-j \sigma \Delta S(\vec{r}, \lambda \vec{f})}\right] d \vec{r}
$$

Then, the absolute square of $O T F_{\text {err }}(\vec{f})$ becomes:

$$
\left|O T F_{e r r}(\vec{f})\right|^{2}=\iint \Omega\left(\vec{r}, \vec{r}^{\prime}, \lambda \vec{f}\right) E\left[e^{-j \sigma \Delta S(\vec{r}, \lambda \vec{f})}\right] E\left[e^{+j \sigma \Delta S\left(\vec{r}^{\prime}, \lambda \vec{f}\right)}\right] d \vec{r} d \vec{r}^{\prime}
$$


where $\Omega\left(\vec{r}, \vec{r}^{\prime}, \lambda \vec{f}\right)$ is defined for simplicity as,

$$
\Omega\left(\vec{r}, \vec{r}^{\prime}, \lambda \vec{f}\right)=\left|O T F_{A}(\vec{f})\right|^{2} \cdot A(\vec{r}) A(\vec{r}-\lambda \vec{f}) A\left(\vec{r}^{\prime}\right) A\left(\vec{r}^{\prime}-\lambda \vec{f}\right) .
$$

On the other hand, the OTF for the ideal telescope, $O T F_{o}(\vec{f})$, is $O T F_{\text {err }}(\vec{f})$ when $\sigma$ becomes 0 .

$$
\left|O T F_{o}(\vec{f})\right|^{2}=\iint \Omega\left(\vec{r}, \vec{r}^{\prime}, \lambda \vec{f}\right) d \vec{r} d \vec{r}^{\prime}
$$

Using its definition in Eq. (2), we obtain the PSSN for this aberration.

$$
P S S N=\left\langle\left\langle E\left[e^{-j \sigma \Delta S(\vec{r}, \lambda \vec{f})}\right] E\left[e^{+j \sigma \Delta S\left(\vec{r}^{\prime}, \lambda \vec{f}\right)}\right]\right\rangle\right\rangle
$$

where $\langle\langle\cdot\rangle\rangle$ defines another operator such that,

$$
\langle\langle\cdot\rangle\rangle \equiv \frac{\iiint \Omega\left(\vec{r}, \vec{r}^{\prime}, \lambda \vec{f}\right)(\cdot) d \vec{r} d \vec{r}^{\prime} d \vec{f}}{\iiint \Omega\left(\vec{r}, \vec{r}^{\prime}, \lambda \vec{f}\right) d \vec{r} d \vec{r}^{\prime} d \vec{f}}
$$

Assuming that the wavefront errors are small, we can expand Eq. (26) in terms of $\sigma$ using the Taylor expansion. Then, Eq. (26) is simplified as,

$$
P S S N=1+a_{1} \sigma+a_{2} \sigma^{2}+\cdots
$$

where $a_{i}$ are the coefficients defined as:

$$
a_{n}=j^{n}\left(\sum_{k=0}^{n} \frac{(-1)^{k}}{k !(n-k) !}\left\langle\left\langle E\left[\Delta S(\vec{r}, \lambda \vec{f})^{k}\right] E\left[\Delta S\left(\vec{r}^{\prime}, \lambda \vec{f}\right)^{n-k}\right]\right\rangle\right\rangle\right)
$$

Note that odd coefficients are all pure imaginary numbers while even coefficients are all real, suggesting that odd orders are all vanishing since the OTF absolute square is a real number. Therefore, Eq. (28) shows that the first dominant term with respect to RMS WFE is the second order and the PSSN is an even function to RMS WFE. This proves $P 3$ in section 2.2. Note also that P3 is valid for deterministic aberration as well as random since Eq. (26) is obtained directly from the definition of the PSSN and $\langle\langle\cdot\rangle\rangle$ is the generalized representation of the operator defined in Eq. (3).

\subsubsection{Relation to Strehl}

We find that the $\alpha$ value in $P 3$ is the negative value of $a_{2}$ in Eq. (29), i.e.

$$
\alpha=-a_{2}=\frac{1}{2}\left\langle\left\langle E\left[\Delta S(\vec{r}, \lambda \vec{f})^{2}\right]+E\left[\Delta S\left(\vec{r}^{\prime}, \lambda \vec{f}\right)^{2}+2 E[\Delta S(\vec{r}, \lambda \vec{f})] \cdot E\left[\Delta S\left(\vec{r}^{\prime}, \lambda \vec{f}\right)\right]\right\rangle\right\rangle\right.
$$

Suppose that the OPD of the aberration is zero-mean and the correlation length of the aberration is zero, i.e. zero-mean white noise. Then, $E[\Delta S(\vec{r}, \lambda \vec{f})]$ becomes 0 . Furthermore,

$$
\begin{aligned}
E\left[\Delta S(\vec{r}, \lambda \vec{f})^{2}\right]=E\left[\Delta S(\vec{r}, \lambda \vec{f})^{2}\right] & =E\left[\left(S(\vec{r})-S(\vec{r}-\lambda \vec{f})^{2}\right]\right. \\
& =\left(E\left[S(\vec{r})^{2}\right]+E\left[S(\vec{r}-\lambda \vec{f})^{2}\right]-2 E[S(\vec{r}) S(\vec{r}-\lambda \vec{f})]\right) \\
& =2
\end{aligned}
$$

Therefore, we find the $\alpha$ value for the white noise aberration.

$$
\alpha=2
$$

This implies that energy spreads out beyond any $r_{o}$ if the aberration is white noise and the PSSN becomes the square of the Strehl. This proves $P_{4}$ in section 2.2 . 


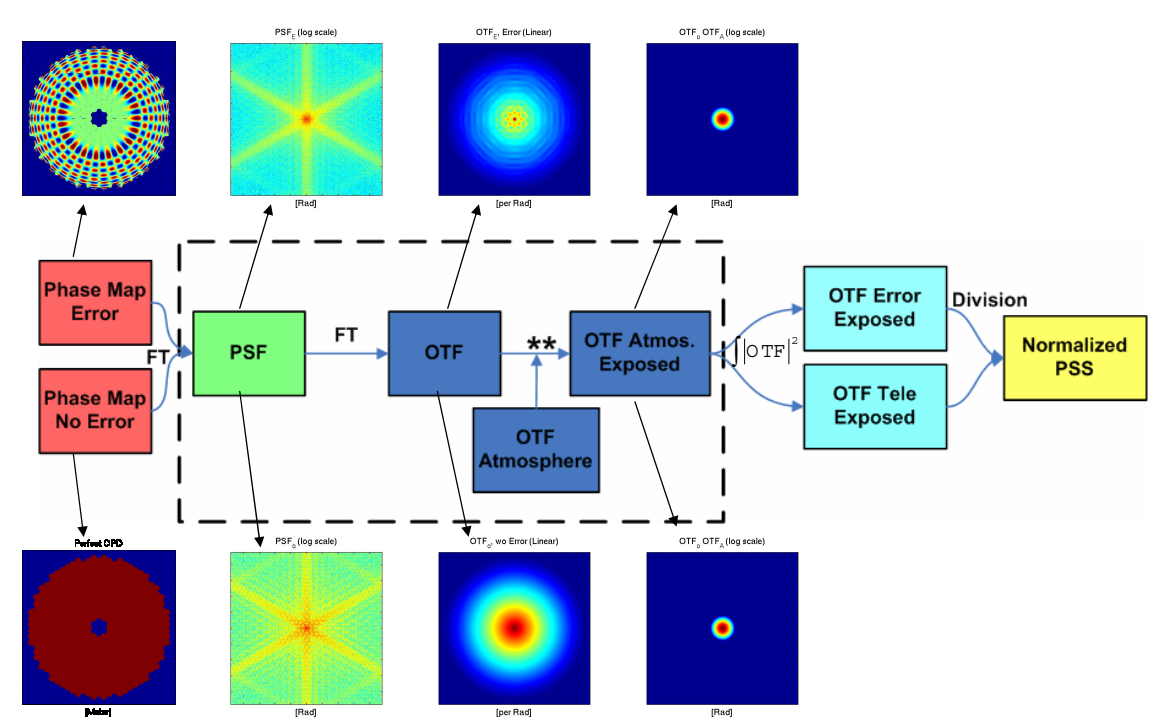

Figure 2. Numerical procedure to calculate PSSN and Zernike mode 800 aberration for example.

\section{NUMERICAL ANALYSIS OF POINT SOURCE SENSITIVITY}

\subsection{Simulation Method}

In order to verify the properties of the PSSN we have investigated in the analytical studies in the previous section, we perform numerical simulations with Zernike aberrations on the TMT pupil. Fig. 2 shows the numerical calculation procedure for the PSSN with an example of Zernike mode* 800 aberration. First, we implement Zernike aberrations in the TMT pupil map. After we obtain their PSFs and OTFs using the fast Fourier transform (FFT) algorithm built in Matlab (The MathWorks, Inc.), we produce the atmosphere exposed OTFs by multiplying the OTFs and atmosphere OTF denoted in Eq. (46). Then, we obtain the PSS err (before normalization as in the numerator in Eq. (2)) in the OTF domain using the Parseval's energy conservation theorem. This computation flow is shown in top of Fig. 2. Likewise as in bottom of Fig. 2, we compute the atmosphere exposed $P S S_{o}$ for ideal telescope (before normalization as in the denominator in Eq. (2)) using an unaberrated OPD map. Finally, we obtain the normalized PSS by dividing $P S S_{\text {err }}$ by $P S S_{o}$.

For the TMT pupil map, we first use the Modeling and Analysis for Controlled Optical Systems (MACOS) software $^{4}$ to define the logical region of the pupil and then employ the gray-pixel approximation $(\mathrm{GPA})^{5}$ in order to simulate the segments' gaps effectively . MACOS is a tool for optical systems analysis developed at the Jet Propulsion Laboratory (JPL) and has been used on projects such as JWST, SIM, TPF, and HST for optical and integrated modeling purposes. MACOS has the desirable capability for our simulation including modeling the gaps between segments and the actual side lengths using a hexagonal coordinate scheme for their placement and running through a Matlab interface, which provides for a vast range of use including high-resolution surface modeling, optical sensitivity calculation, and wavefront sensing and control simulation. We use a $1 / 64 \mathrm{~m}$ per pixel sampling grid with $2048 \times 2048$ pixels across the pupil, the Nyquist sampling for FFT, an atmosphere $r_{o}$ of $200 \mathrm{~mm}$, and the wavelength of $500 \mathrm{~nm}$ for the numerical simulation parameters.

\subsection{Point Source Sensitivity for Zernike Aberrations}

Zernike aberrations of mode 4, 200, 400, 800 on the TMT pupil are shown in Fig. 3(a) to Fig. 3(d), respectively. Using the numerical method, we compute the PSSN with respect to the RMS WFE and the results are shown in Fig. 4(a). The simulated RMS WFE ranges from 0 to $160 \mathrm{~nm}$ and The wavelength and atmosphere $r_{o}$ considered are $500 \mathrm{~nm}$ and $200 \mathrm{~mm}$, respectively. For all aberrations, the PSSN is 1 when the RMS WFE is zero and decreases as RMS WFE increases. The PSSN decreases faster for higher Zernike aberration as expected. The $\log -\log$ scale plots of 1-PSSN is shown in Fig. 4(b). Note that the plots in Fig. 4(b) are close to linear lines and the ratio between the $\mathrm{x}$ and $\mathrm{y}$ axis is 2, verifying the P3 in Eq. (7).

${ }^{*}$ All Zernike mode in this paper refers to Zernike Noll Modes. ${ }^{3}$ 


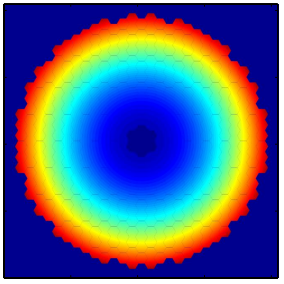

(a) Mode 4

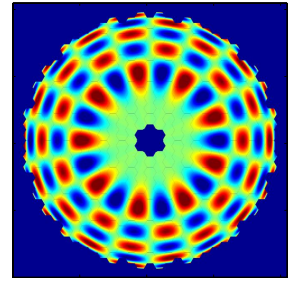

(b) Mode 200

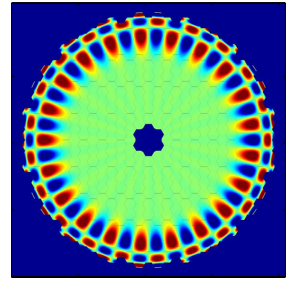

(c) Mode 400

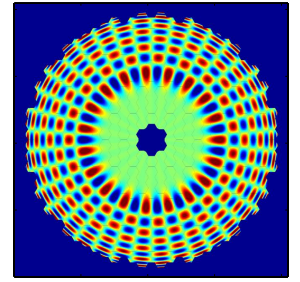

(d) Mode 800

Figure 3. Example Zernike Modes on TMT pupil

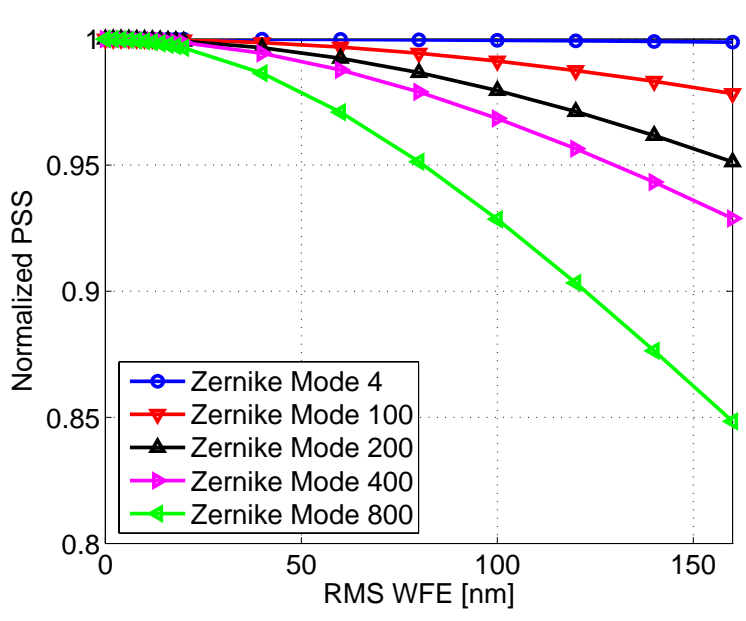

(a) PSSN vs RMS

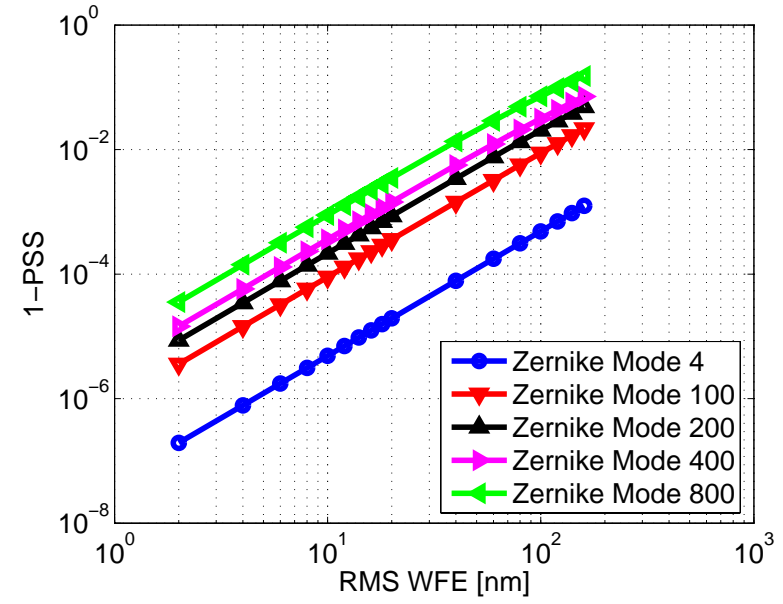

(b) PSSN vs RMS (loglog)

Figure 4. Numerically calculated PSSN for Zernike 4, 100, 200, 400 and 800 modes with respect to the RMS WFE. The simulated RMS WFE ranges from 0 to $160 \mathrm{~nm}$. (a) PSSN plot in linear scale and (b) 1-PSSN plot in log log scale.

In addition, we have performed similar simulations to other Zernikes and we compute the $\alpha$ values in Eq. (7) and summarize the results in Table. 1. From the results, we observe that the $\alpha$ values of lower azimuthal aberrations are larger in the same radial Zernike mode. We also find that the $\alpha$ value for the white noise is close to 2 as we expect in $P 4$ in Eq. (8).

In order to verify the multiplicative feature of PSSN (P2), we first obtain combined OPDs by adding all OPDs which have same RMS WFE for Zernikes shown in Fig. 4 and then calculate the combined PSSNs and RMS WFEs for the combined OPDs. We also obtain multiplied PSSNs by multiplying the PSSN values for all considered Zernikes with same RMS WFE. Then, we compare the combined PSSNs to multiplied PSSNs. Fig. 5(a) shows the simulation results. The difference between combined and multiplied PSSN values is small when the RMS WFE is small (or the multiplied PSSN is close to 1) and increases as the RMS WFE increases (or the multiplied PSSN becomes worse). The $\mathrm{x}$ axis in Fig. 5(a) represents the computed RMS WFE for the combined OPDs and they are similar to the RSSed RMS WFE due to the orthogonality of Zernikes. However, they disagree with a small amount due to hexagonal outline of the TMT pupil and we ignore this discrepancy.

Fig. 5(b) shows that the difference, $\Delta_{N}$, between the combined and multiplied PSSN with respect to the multiplied PSSN values. Zernike aberrations are deterministic and shift-variant while our analytical boundary of $\Delta_{A}$ in Eq. (18) is approximated for random shift-invariant errors, the numerical $\Delta_{N}$ cannot be directly compared to the analytical boundary $\Delta_{A}$. Nonetheless, we compare the numerical $\Delta_{N}$ to the analytical boundary $\Delta_{A}$ using Eq. (18) from the individual PSSN values in Fig. 5(b). Even though the numerical $\Delta_{N}$ is larger than the analytical boundary $\Delta_{A}$, we observe that $\Delta_{N}$ is close to 0.01 when if the PSSN is larger than 0.9 . 


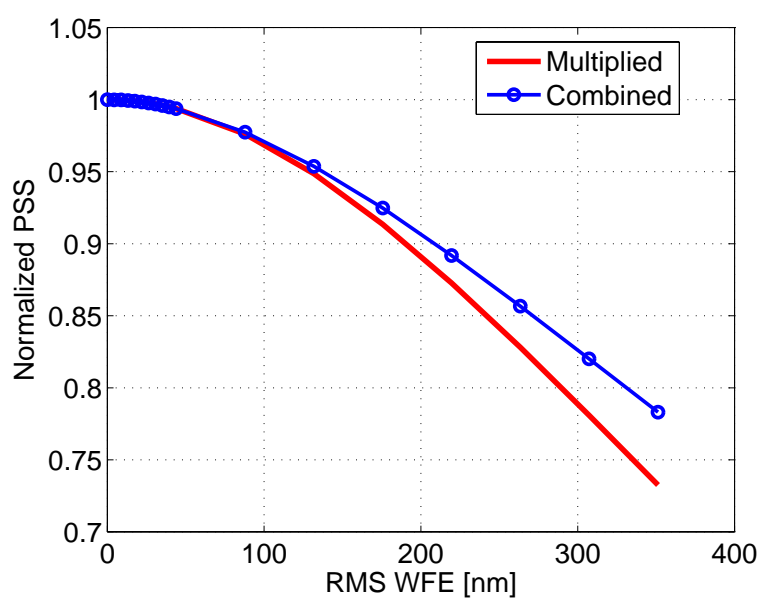

(a) Combined and Multiplied PSSN

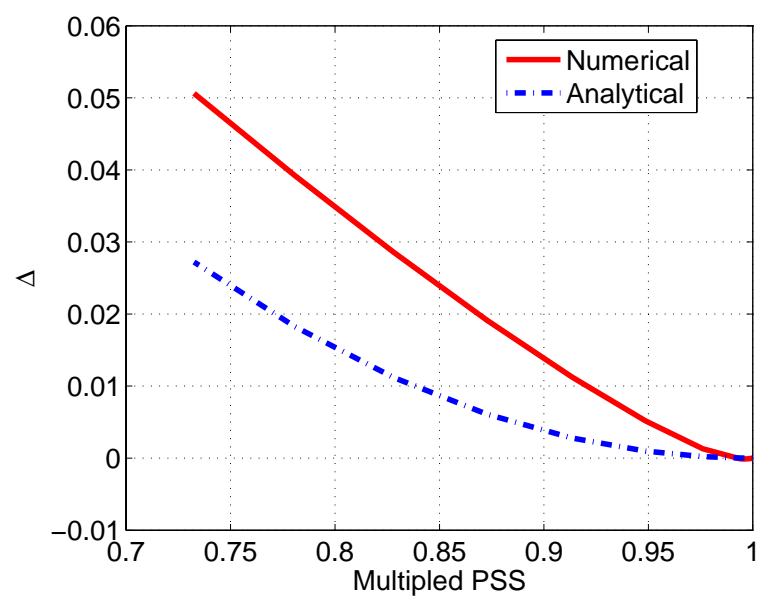

(b) Multiplied PSSN vs $\Delta$

Figure 5. (a) Combined and Multiplied PSSN for Zernikes considered in Fig. 4. (b) Difference $\Delta_{N}$ between combined and multiplied PSSN with respect to the multiplied PSSN. Even though comparison is not valid since the Zernike aberrations are deterministic, we compare the difference to analytical difference boundary $\Delta_{A}$ using Eq. (18). We observe that $\Delta_{N}$ is still close to 0.01 when if the PSSN is larger than 0.9 .

For a more quantitative verification of the analytical boundary $\Delta_{A}$, we compare numerical $\Delta_{N}$ and analytical boundary $\Delta_{A}$ for the TMT M1 primary errors as shown in Fig. 6 . The numerically calculated $\Delta_{N}$ is from intensive studies done by Nissly, et. al., ${ }^{1}$ and the analytical boundary $\Delta_{A}$ is from Eq. (18) with individual PSSN values from Nissly, et. al. Since the TMT M1 primary errors includes random errors which can have shift-invariant characteristics, $\Delta_{N}$ and $\Delta_{A}$ match better than when compared to Zernike aberrations. As seen in Fig. 6, the numerically obtained $\Delta_{N}$ are within the analytical boundary of $\Delta_{A}$ for most zenith angles, supporting that our analytical studies and approximations are valid for the practical errors in the TMT system engineering.

Table 1. Alpha Table

\begin{tabular}{|c|c|c|r|}
\hline Zernike Mode & Radial Mode & Azimuthal Mode & $\alpha\left[/ \mathrm{rad}^{2}\right]$ \\
\hline 4 & 2 & 0 & $3.086 \mathrm{e}-04$ \\
5 & 2 & 2 & $1.488 \mathrm{e}-04$ \\
6 & 2 & 2 & $1.488 \mathrm{e}-04$ \\
7 & 3 & 1 & $5.573 \mathrm{e}-04$ \\
8 & 3 & 1 & $5.575 \mathrm{e}-04$ \\
9 & 3 & 3 & $2.978 \mathrm{e}-04$ \\
10 & 3 & 3 & $2.939 \mathrm{e}-04$ \\
56 & 10 & 0 & $5.925 \mathrm{e}-03$ \\
66 & 10 & 10 & $2.598 \mathrm{e}-03$ \\
211 & 20 & 0 & $1.912 \mathrm{e}-02$ \\
231 & 20 & 20 & $9.370 \mathrm{e}-03$ \\
821 & 40 & 0 & $7.009 \mathrm{e}-02$ \\
861 & 40 & 40 & $3.296 \mathrm{e}-02$ \\
1831 & 60 & 0 & $4.862 \mathrm{e}-01$ \\
1891 & 60 & 60 & $7.143 \mathrm{e}-02$ \\
3082 & 78 & 0 & $1.403 \mathrm{e}+00$ \\
3160 & 78 & 78 & $1.038 \mathrm{e}-01$ \\
White Noise & NA & NA & $1.962 \mathrm{e}+00$ \\
\hline
\end{tabular}




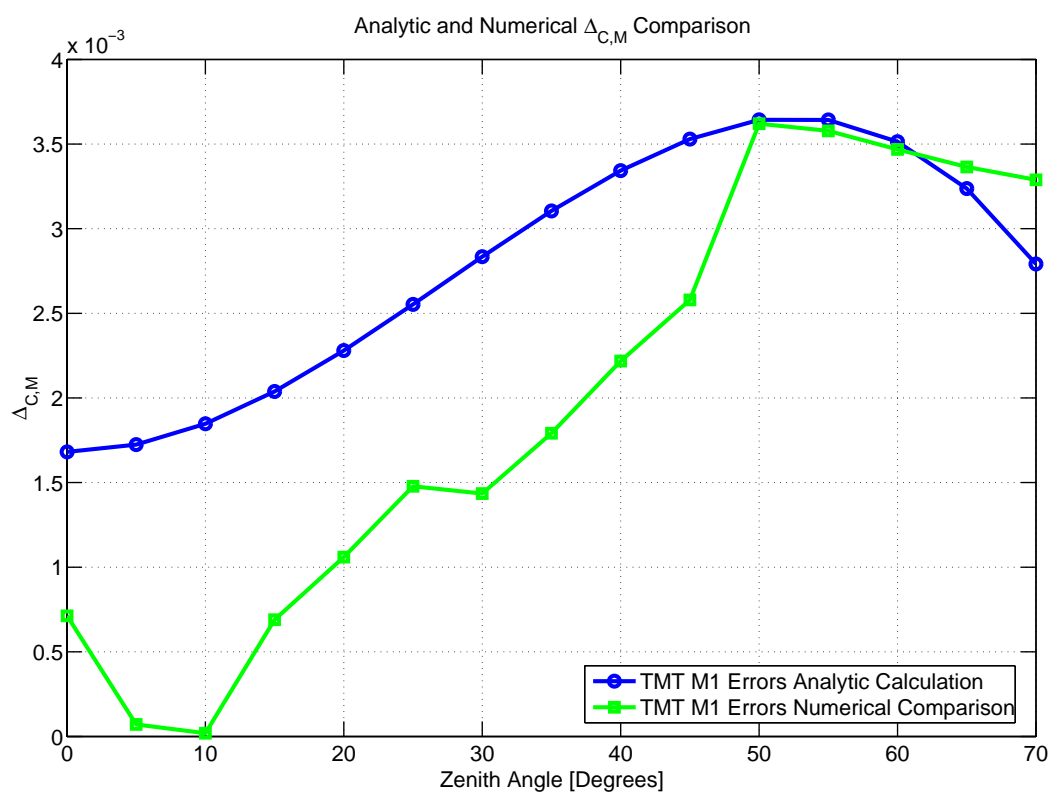

Figure 6. Numerical difference $\Delta_{N}$ and analytical difference boundary $\Delta_{A}$ between combined and multiplied PSSN for TMT M1 primary errors. Two plots are obtained using two independent methods. Numerically calculated $\Delta_{N}$ is from intensive studies done by Nissly, et. al., ${ }^{1}$ and the analytical boundary $\Delta_{A}$ is from Eq. (18) with individual PSSN values from Nissly, et. al.

\section{SUMMARY}

Among other science metrics, the normalized PSS is distinguished in that it includes atmosphere information by appropriately weighting telescope errors in terms of atmosphere. Therefore, we believe that the normalized PSS is truly a measure of the science loss for seeing limited instruments. Furthermore, it has a multiplicative feature when combining multiple error sources. In this paper, we find that the multiplicative feature is valid within a few percent (within $1 \%$ for shift-invariant errors) when the science loss of all combined errors is smaller than $10 \%$, i.e., the combined PSSN is larger than $90 \%$. Since TMT has a goal that the telescope should not introduce more than a $10 \%$ loss of science, we believe that this metric is useful for optical designing and budgeting errors of the TMT.

\section{APPENDIX A.}

For aberrations whose the OPD has random and shift-invariant characteristics, prove that the OTF $F_{\text {err }}$ (timeaveraged OTF of an aberrated telescope exposed to the atmosphere) can be represented as a multiplication of $O T F_{o}$ and $O T F_{e}$, where $O T F_{o}$ is the time-averaged OTF of the ideal telescope exposed to the atmosphere and $O T F_{e}$ are the time-averaged $\mathrm{OTF}$ due to the telescope aberrations only. i.e.

$$
O T F_{\text {err }}(\vec{f})=O T F_{e}(\vec{f}) \cdot O T F_{o}(\vec{f})
$$

\section{[Proof.]}

The instantaneous or static PSF (before exposed to atmosphere) due to aberrated OPD and telescope can be represented as,

$$
P S F_{e+t}(\vec{\theta})=\left|\int_{r} A(\vec{r}) e^{-j k(O P D(\vec{r})} e^{j \vec{\theta} \vec{r}} d \vec{r}\right|^{2}
$$

where $\vec{r}$ and $d \vec{r}$ are the two-dimensional coordinates for the OPD (typically in meter units), $k$ is the wavenumber defined as $2 \pi / \lambda, \lambda$ is the wavelength, $A(\vec{r})$ is the real, nonnegative telescope pupil function normalized with 
$\int A^{2}(\vec{r}) d \vec{r}=1$. The subscripts $e$ and $t$ in $P S F_{e+t}$ represent that the PSF is due to aberrated OPD and telescope, respectively. Using the Fourier transformation properties and integration properties, the OTF of $P S F_{e+t}$ (before exposed to atmosphere) can be obtained and simplified as the same equation, Ellerbroek derived. ${ }^{6}$

$$
O T F_{e+t}(\vec{f})=\int_{r} A(\vec{r}) A(\vec{r}-\lambda \vec{f}) e^{-j k(O P D(\vec{r})-O P D(\vec{r}-\lambda \vec{f}))} d \vec{r}
$$

where $\vec{f}$ is the coordinate for the OTF space (typically in cycles per radian). We obtain the OTF $F_{\text {err }}$, timeaveraged OTF of the aberrated telescope exposed to the atmosphere if we consider exposed OTF of atmosphere, $O T F_{A}$.

$$
O T F_{\text {err }}(\vec{f})=O T F_{A}(\vec{f}) \cdot \int_{r} A(\vec{r}) A(\vec{r}-\lambda \vec{f}) E\left[e^{-j k(O P D(\vec{r})-O P D(\vec{r}-\lambda \vec{f})}\right] d \vec{r}
$$

Note that $O T F_{A}$ is independent to the pupil coordinates $\vec{r}$, and the operator, $E[\cdot]$, denotes the time averaged (or statistic averaged) of the aberration. If the OPD has the random shift-invariant characteristics, i.e. $E\left[e^{-j k(O P D(\vec{r})-O P D(\vec{r}-\lambda \vec{f})}\right]$ is only a function of $\lambda \vec{f}$, the $O T F_{\text {err }}$ is further simplified as,

$$
O T F_{\text {err }}(\vec{f})=O T F_{e}(\vec{f}) \cdot O T F_{A}(\vec{f}) \cdot O T F_{t}(\vec{f})
$$

where $O T F_{e}$ is the time-averaged $\mathrm{OTF}$ due to the telescope aberration only and $O T F_{t}$ is the deterministic OTF of the ideal telescope, i.e.

$$
\begin{aligned}
O T F_{e}(\vec{f}) & =E\left[e^{-j k(O P D(\vec{r})-O P D(\vec{r}-\lambda \vec{f})}\right] \\
O T F_{t}(\vec{f}) & =\int_{r} A(\vec{r}) A(\vec{r}-\lambda \vec{f}) d \vec{r}
\end{aligned}
$$

Note that $O T F_{o}$ is equivalent to $O T F_{A} O T F_{t}$, therefore we prove Eq. (33).

\section{APPENDIX B.}

Assume A3 is valid and two different errors generate $\epsilon_{1}$ and $\epsilon_{2}$, respectively and individual PSSN values are $P S S_{1}$ and $P S S_{2}$, respectively. Using the Taylor expansion, prove that

$$
\Delta_{1,2} \equiv<\epsilon_{1} \epsilon_{2}>-<\epsilon_{1}><\epsilon_{2}>\quad \approx 1.2119\left(1-P S S_{1}\right)\left(1-P S S_{2}\right)
$$

\section{[Proof.]}

First, we approximate individual $\epsilon_{i}(x, y)$ as,

$$
\epsilon_{i}(x, y)=a_{1} x+b_{2} y+a_{2} x^{2}+b_{2} x^{2}+c_{1,1} x y+\cdots
$$

where the coefficients, $a_{k}, b_{k}$, and $c_{i, j}$ are $\left.\frac{1}{k !} \frac{\partial^{k} \epsilon}{\partial x^{k}}\right|_{(0,0)},\left.\frac{1}{k !} \frac{\partial^{k} \epsilon}{\partial y^{k}}\right|_{(0,0)}$, and $\left.\frac{i+j}{(i+j) !} \frac{\partial^{i+j} \epsilon}{\partial^{i} x \partial^{j} y}\right|_{(0,0)}$, respectively. Since the OTF has a Fourier transformation relation to the PSF and the PSF is a real positive valued function, according to the Fourier transform property, OTF (hence $\epsilon$ ) are even functions. Therefore, odd orders are all dropped in Eq. (40). With the further assumption that errors are isotropic in the $\vec{f}$ domain, i.e. , $a_{k}=b_{k}=\alpha_{k}$ and $c_{i, j}=0$ and if we approximate Eq. (40) up to second order terms,

$$
\epsilon_{i}(r) \approx \alpha_{2} r^{2}
$$

Therefore, $\Delta_{1,2}$ can be approximated as,

$$
\Delta_{1,2}=p_{2} q_{2}\left(<r^{4}>-<r^{2}>^{2}\right)
$$

where $p_{2}$ and $q_{2}$ are $\alpha_{2}$ for error 1 and error 2, respectively. Since we know the individual PSSN values,

$$
\begin{aligned}
& 1-P S S_{1}=<\epsilon_{1}>=p_{2}<r^{2}> \\
& 1-P S S_{2}=<\epsilon_{2}>=q_{2}<r^{2}>
\end{aligned}
$$


Eq. (42) becomes,

$$
\Delta_{1,2} \approx \frac{\left(<r^{4}>-<r^{2}>^{2}\right)}{<r^{2}>^{2}}\left(1-P S S_{1}\right)\left(1-P S S_{2}\right)
$$

Since the atmosphere OTF is well defined, $\frac{\left(\left\langle r^{4}\right\rangle-\left\langle r^{2}\right\rangle^{2}\right)}{\left\langle r^{2}\right\rangle^{2}}$ is a scalar value that can be computed to a finite value. From Appendix C, we find the constant as 1.2119, hence, we prove Eq. (39).

\section{APPENDIX C.}

Compute and prove

$$
\frac{\left(<r^{4}>-<r^{2}>^{2}\right)}{<r^{2}>^{2}}=1.2119 \cdots
$$

[Proof.]

The OTF of the long exposure atmosphere is defined as,

$$
O T F_{A}(r)=\exp \left(-3.44\left(\frac{r}{r_{o} / \lambda}\right)^{5 / 3}\right)
$$

Using integral properties and definition defined in Eq. (4), $\left\langle r^{n}>\right.$ becomes:

$$
<r^{n}>=\left(\frac{r_{o}}{\lambda}\right)^{n} r^{n}
$$

where $n=1,2, \cdots, r^{\bar{n}}=\frac{\int_{\infty} r^{n} w(r) r d r}{\int_{\infty} w(r) r d r}$, and $w(r)=\exp \left(-2 \times 3.44 \times r^{5 / 3}\right)$. We calculate $\overline{r^{n}}$ analytically to obtain,

$$
\frac{\left(<r^{4}>-<r^{2}>^{2}\right)}{<r^{2}>^{2}}=\frac{\left(\overline{r^{4}}-\bar{r}^{2}\right)}{\bar{r}^{2}}=1.2119 \cdots
$$

\section{ACKNOWLEDGMENTS}

This research was carried out in part at the Jet Propulsion Laboratory, California Institute of Technology, and was sponsored by the California Institute of Technology and the National Aeronautics and Space Administration. The authors gratefully acknowledge the support of the TMT partner institutions. They are the Association of Canadian Universities for Research in Astronomy (ACURA), the California Institute of Technology and the University of California. This work was supported as well by the Gordon and Betty Moore Foundation, the Canada Foundation for Innovation, the Ontario Ministry of Research and Innovation, the National Research Council of Canada, the Natural Sciences and Engineering Research Council of Canada, the British Columbia Knowledge Development Fund, the Association of Universities for Research in Astronomy (AURA) and the U.S. National Science Foundation.

\section{REFERENCES}

1. C. Nissly, B. Seo, M. Troy, G. Angeli, J. Angione, I. Crossfield, B. Ellerbroek, L. Gilles, and N. Sigrist, "High-resolution optical modeling of the Thirty Meter Telescope for systematic performance trades," Proc. SPIE 7017-30, June 2008.

2. I. R. King, "Accuracy of measurement of star images on a pixel array," Publications of Astronomical Society of the Pacific 95, pp. 163-168, Feb 1982.

3. R. J. Noll, "Zernike polynomials and atmospheric turbulence," J. Opt. Soc. Am. 66, p. 207, 1976.

4. D. Redding et al., "MACOS manual (modeling and analysis for controlled optical systems)," NASA JPL D-9816, internal document 5, 1999.

5. M. Troy and G. Chanan, "Diffraction effects from giant segmented-mirror telescopes," Applied Optics 42, pp. 3745-3753, July 2003.

6. B. Ellerbroek, "OTF and structure function methods for (Seeing-Limited) TMT error budgets," Project Communication TMT.SEN.PRE.05.002.REL01, April 2005. 